\title{
Formation of the Nonlinear Dynamic Hologram in Clear Organic Fluids
}

\author{
O. Ormachea and A. L. Tolstik \\ Belarussian State University, ul. Leningradskaya 14, Minsk, 220080 Belarus \\ e-mail: ormachea@bsu.by; tolstik@bsu.by
}

\begin{abstract}
The recording of dynamic holograms during multiwave interactions in clear organic fluids (ethanol, acetone, dioxan, etc.) is investigated. It is shown that the fifth-order nonlinearities and higher become activated along with the cubical nonlinearity under the intense laser excitation $\left(\sim 100 \mathrm{MW} \mathrm{cm}{ }^{-2}\right)$ in the near-IR spectral range, which allows visualization of the IR images using different orders of diffraction.
\end{abstract}

DOI: $10.3103 / \mathrm{S} 1062873807010327$

\section{INTRODUCTION}

Colored and clear organic fluids are widely used in contemporary laser physics as nonlinear media [1,2]. A light-induced change in the optical properties of nonlinear media in the interference field of the interacting waves leads to recording of the dynamic diffraction gratings (holograms). Their formation can be associated with variation in the refractive index and the coefficient of the absorption during molecule transition to higher energy states, and with the thermal nonlinearity caused by the media heating.

Our work describes a general approach to analyzing processes of multiwave interaction of light beams in clear organic fluids when there are higher-order nonlinearities caused by the nonlinear character as the thermal dynamic gratings form.

Typically, recording of the nonlinear dynamic hologram during multiwave interactions is realized in resonance media (pigment solutions, crystals with color centers, etc.) in which the nonlinearities of the fifth order and higher are induced due to the effect of absorption saturation [3]. The nonlinear dependence of lightinduced variation in the refractive index of resonance media on the intensity under these conditions leads to the distortion in the profile of the element of the holographic grating, which ceases to be sinusoidal. For analysis of the diffraction characteristics of such dynamic structures, one can use Fourier decomposition (expansion) of the spatial distribution of the nonlinear response of the media along the spatial harmonics of the grating [4]. The dispersion at different harmonics of the grating determines the diffraction in the second and higher orders. Provided that the condition of threedimensionality of the gratings of the dynamic holograms is satisfied, the angular selectivity of the grating allow us to individually restore the waves diffracted into the different orders, by varying the frequency [4] or the direction of the propagation of the readout beam [5].

\section{EXPERIMENTAL REALIZATION OF MULTIWAVE INTERACTION IN CLEAR ORGANIC FLUIDS}

Experimental investigation of the diffraction of the laser radiation on the thermal dynamic gratings was conducted using frequency nondegraded multiwave interaction (Fig. 1). An yttrium-aluminum garnet laser, operating in the active modulation of the quality mode (pulse duration $\tau=15 \mathrm{~ns}$ ), was used as a radiation source. Spectrum divider (3) and mirrors (8) and (9) formed parallel propagating signal $E_{S}$ and reference $E_{1}$ waves at the primary generation frequency of the laser (wavelength $\lambda=1064 \mathrm{~nm}$ ). The recording of the dynamic hologram was performed during interference of the signal and reference waves in the container with organic fluid (7). Clear ethanol, acetone, dioxan, isopropanol, and dichlorethan, possessing low absorption in the near-IR spectrum (container width $3 \mathrm{~mm}$ ), were used as the organic fluid. Mirror (4) was used to direct readout wave $E_{2}$ at the doubled frequency $(\lambda=532 \mathrm{~nm})$ in the direction opposite to the pumping hologram waves. Mirror (4) could be moved, allowing the change of the readout angle. Detection system (5), (11) was used for measuring the energetic efficiency of the multiwave interaction. Filters (6) and (10) were used to limit radiation power. The phase synchronization condition for the multiwave interaction $\mathbf{k}_{D}=M \mathbf{k}_{1}-M \mathbf{k}_{S}+$

$\mathbf{k}_{2}\left(\mathbf{k}_{i}\right.$ are the wave vectors of the interacting waves and $M$ is the order of the diffraction) defines the direction of the readout wave propagation [3]. Typical interaction 
geometry is presented in Fig. 2. The first case (fourwave interaction, Fig. 2a) corresponds to realization of the recording of the dynamic holograms on cubical nonlinearity, when the polarization responsible for the generation of the diffracted wave $E_{D}$ with doubled frequency $2 \omega$ is $P(2 \omega) \sim \chi^{(3)} E_{1}(\omega) E_{S}^{*}(\omega) E_{2}(2 \omega)$. Corresponding condition of the phase synchronization $\mathbf{k}_{D}=$ $\mathbf{k}_{1}-\mathbf{k}_{S}+\mathbf{k}_{2}$ requires the readout wave $E_{2}$ to be rotated by $\beta \approx \alpha / 4$ relative to the direction of the reference wave $E_{1}(\alpha$ is the angle between the waves recording hologram). In the six-wave interaction (Fig. 2b), the nonlinear polarization becomes $P \sim \chi^{(5)}\left(E_{1} E_{S}^{*}\right)^{2} E_{2}$ and the condition of the phase synchronization $\mathbf{k}_{D}=2 \mathbf{k}_{1}-2 \mathbf{k}_{S}+$ $\mathbf{k}_{2}$ is realized at the opposite direction of the reference and the readout waves propagation $\left(\beta=0,2 \mathbf{k}_{1}+\mathbf{k}_{2}=0\right)$. In this case the diffracted wave also propagates in the direction opposite to the signal wave regardless of the interaction geometry $\left(\mathbf{k}_{D}=-2 \mathbf{k}_{S}\right)$.

The realization of the multiwave interaction in the clear organic liquids is attributed to the activation of the thermal nonlinearity due to the absorption at a wavelength of $\lambda=1064 \mathrm{~nm}$ (absorption coefficient $k \leq$ $\left.0.1 \mathrm{~cm}^{-1}\right)$. The value of the thermal change of the refractive index can be estimated by the known equation [6]

$$
\Delta n_{T}=(d n / d T) \Delta T=(d n / d T) k I \tau / C_{\rho},
$$

where $d n / d T$ is thermooptical coefficient, $I$ is intensity of the interference field, $\tau$ is pulse duration, and $C_{\rho}$ is heat capacity of the unit of volume. As can be seen from this equation, the low value of the absorption coefficient can be compensated by the increase in the intensity of the pumping waves. The experimental studies of the efficiency of the recording of the dynamic holograms in the various organic fluids at $\leq 300 \mathrm{MW} \mathrm{cm}$ intensity of the interacting waves have shown that the four-wave interaction, caused by the recording of the thermal dynamic gratings, is realized in all considered (utilized, tested) media (ethanol, acetone, dioxan, isopropanol, and dichlorethan). The highest values of the diffraction efficiency of the dynamic hologram $\xi=I_{D} / I_{2}$ (up to $15 \%$ ) were obtained by using ethanol. Its dependences of the diffraction efficiency versus the intensity of the pumping waves $\left(I_{1}=I_{S}=I\right)$ are provided in Fig. 3a. As expected, in accordance with the nonlinear polarization $P \sim \chi^{(3)} E_{1} E_{S}^{*}(\omega) E_{2}$, the increase in intensity leads to an almost quadratic increase in the diffraction efficiency. Similar results were also obtained for isopropanol, characterized by close thermooptical properties. On the other hand, the values of the diffraction efficiency for dioxan were several times lower, and for the acetone and the dichlorethan, lower by several

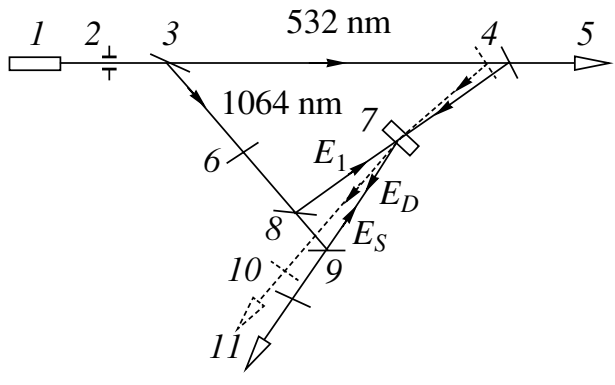

Fig. 1. Schematic of the experimental setup. (1) YttriumAluminum garnet laser, (2) diaphragm, (3) spectrum divider, $(4,8$, and 9$)$ mirrors, $(6,10)$ light filters, $(5,11)$ registration system, and (7) container with organic fluid.
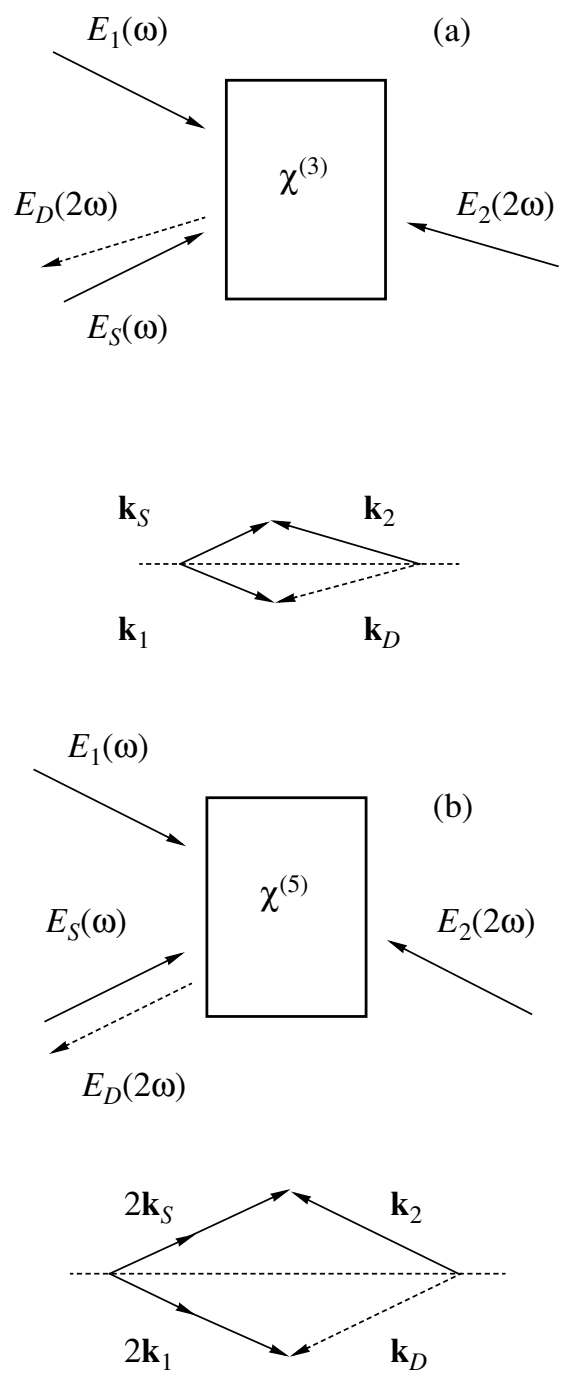

Fig. 2. The schemes of the frequency nondegenerated (a) four- and (b) six-wave interactions and corresponding diagrams of the wave vectors. 

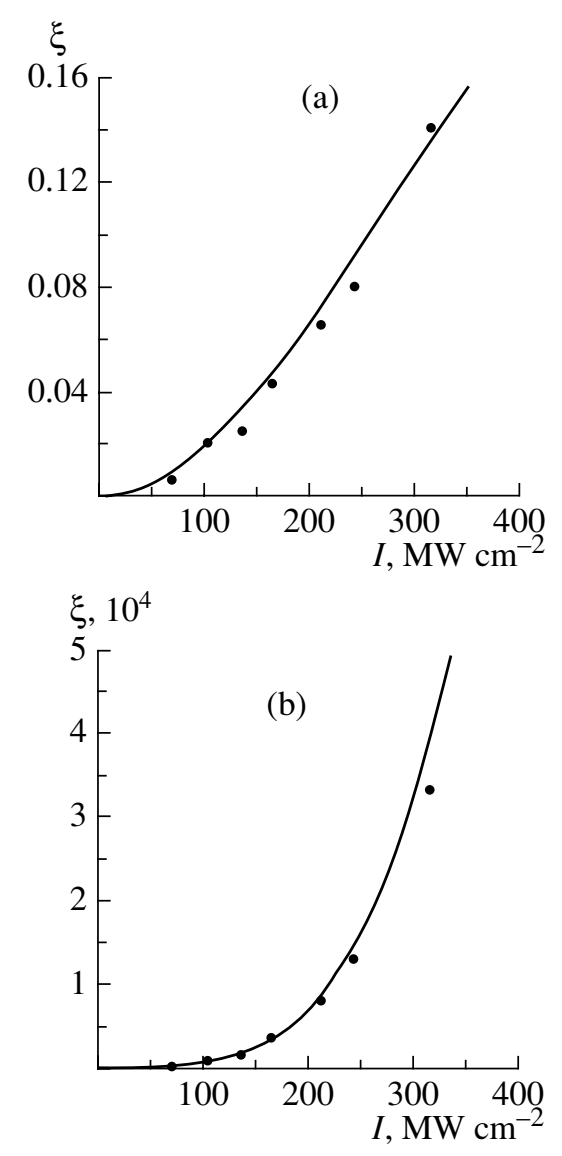

Fig. 3. Diffraction efficiency $\xi$ versus the intensity of the pumping waves for (a) four- and (b) six-wave interaction at $I_{2}=12 \mathrm{MW} \mathrm{cm}^{-2}$ intensity of the readout wave.

orders of magnitude. The most interesting observation is the realization of the six-wave interaction (Fig. 3b), which occurs with the activation of fifth-order nonlinearity. The confirmation of realization of the six-wave interaction, in addition to the geometry of the interaction, is the fourth degree of the dependence of the diffraction efficiency on the intensity of the pumping waves (nonlinear polarization $\left.P \sim \chi^{(5)}\left(E_{1} E_{S}^{*}\right)^{2} E_{2}\right)$. The six-wave interaction was observed in ethanol, isopropilen and dioxan, and, similarly to the four-wave interaction, the values of the diffraction efficiency were close for ethanol and isopropanol, but were several times smaller for dioxan.

Note that previously, when used as a nonlinear pigment solution media (e.g., [3, 4]), the six-wave interaction was realized via the effect of absorption saturation. However, the absorption coefficient of the organic fluids remained constant throughout the experiment. The appearance of the higher-order nonlinearities in this case can be explained by the dependence of the temperature on such parameters, present in the equation (1), as the heat capacity of the unit of volume and the thermooptical coefficient, which were considered constant in all previous analyses. The experimentally obtained values of the diffraction efficiency $\xi \sim 10^{-3}-10^{-4}$ are comparable to the values obtained using polymethin pigments [7] and exceed by three orders of magnitude the values obtained in [8] in the six-wave interaction in polymers using the resonance two-photon absorption mechanism.

\section{THEORETICAL MODEL OF THE NONDEGRADED MULTIWAVE INTERACTION}

In order to give a theoretical description of the multiwave interaction process, let us use the known approximation of the slowly varying amplitudes, which allows us to write a system of shortened wave equations for interacting light fields [3, 4]

$$
\begin{gathered}
\frac{\partial E_{1, S}}{\partial r}=i \frac{2 \pi \omega}{c n_{0}}\left(E_{1, s} \chi_{0}(\omega)+E_{S, 1} \chi_{ \pm 1}(\omega)\right), \\
\frac{\partial E_{2, D}}{\partial r}=-i \frac{4 \pi \omega}{c n_{0}}\left(E_{2, D} \chi_{0}(2 \omega)+E_{D, 2} \chi_{\mp M}(2 \omega)\right) .
\end{gathered}
$$

Here $n_{0}$ is initial value of the refractive index, $\chi_{m}$ are terms of the decomposition of the nonlinear media sensitivity into the spatial Fourier series over the harmonics of the dynamic grating $\mathbf{K}=\mathbf{k}_{1}-\mathbf{k}_{S}$ :

$$
\left.\chi_{m}=\frac{1}{2 \pi} \int_{-\pi}^{\pi} \chi_{n l} \exp [-i m(\mathbf{K} \cdot \mathbf{r}))\right] d(\mathbf{K} \cdot \mathbf{r}),
$$

where the nonlinear sensitivity $\chi_{n l}=n_{0} \Delta n_{T} / 2 \pi$ is associated with the thermal change of the refractive index (1).

The specific terms of the decomposition $\chi_{m}$ are determined by the spectroscopic characteristics of a given media. Considering the experimental realization of the six-wave interaction, we can conclude that the thermooptical characteristics are not constant, but are dependent on the temperature, leading to the saturation character of the thermal nonlinearity, which can be written as

$$
\Delta n_{T}=\frac{\kappa a_{T} I}{1+\alpha I},
$$

where $\kappa=\lambda k / 4 \pi$ is extinction coefficient and $a_{T}=$ $\frac{2 \omega}{c}\left(\frac{d n}{d T}\right) \frac{\tau}{C_{\rho}}, \alpha$ is the nonlinearity parameter determined by the dependence of the thermo-optical characteristics on the temperature.

Taking into account Eq. (5), the terms of the Fourier expansion $\chi_{m}$ (4) can be represented as

$$
\chi_{0}=\frac{n_{0} \kappa}{2 \pi}\left[i+\frac{a_{T}}{\alpha}\left(1-\frac{1}{A}\right)\right]
$$




$$
\begin{aligned}
& \chi_{ \pm 1}=\frac{n_{0} \kappa}{2 \pi}\left[\frac{2 a_{T} \sqrt{I_{1} I_{S}}}{A\left(1+A+\alpha\left(I_{1}+I_{S}\right)\right)}\right] \\
& \times \exp \left[ \pm i\left(\varphi_{1}-\varphi_{S}\right)\right] \text {, } \\
& \chi_{ \pm 2}=\frac{n_{0} \kappa}{2 \pi}\left[\frac{-4 a_{T} \alpha I_{1} I_{S}}{A\left(1+A+\alpha\left(I_{1}+I_{S}\right)\right)^{2}}\right] \\
& \times \exp \left[ \pm i 2\left(\varphi_{1}-\varphi_{S}\right)\right] \\
& \chi_{ \pm M}=\frac{n_{0} \kappa}{2 \pi}\left(-\frac{a_{T}}{\alpha}\right) \frac{\left(-2 \alpha \sqrt{I_{1} I_{S}}\right)^{M}}{A\left(1+A+\alpha\left(I_{1}+I_{S}\right)\right)^{M}} \\
& \times \exp \left[ \pm i M\left(\varphi_{1}-\varphi_{S}\right)\right],
\end{aligned}
$$

where $A=\left(1+2 \alpha\left(I_{1}+I_{S}\right)+\alpha^{2}\left(I_{1}-I_{S}\right)^{2}\right)^{1 / 2}$.

It follows from Eqs. (3) and (9) that, during the restoration of the dynamic hologram at the doubled frequency in the case of the flat reference and readout waves $\left(M \varphi_{1}+\varphi_{2}=\right.$ const $)$, the phase of the diffracted wave $\varphi_{D}=-\varphi_{S}$ under diffraction into the first order (four-wave interaction) and $\varphi_{D}=-2 \varphi_{S}$ (diffraction into the second-order, six-wave interaction). In the latter case the wave $E_{D}$ has frequency $2 \omega$ and propagates in the opposite direction to the signal wave $E_{S}$. Doubled conjugate phase at the simultaneous doubling of the frequency of the signal wave means that both waves $\left(E_{S}\right.$ and $E_{D}$ ) have coinciding surfaces of the wave front at the opposite directions of propagation (the effect of the reversed wave front with simultaneous doubling of the light wave frequency [7]).

The solution of differential equations system (2), (3) allows us to describe the above experimental setup, which realizes the recording of the dynamic holograms at the primary laser generation frequency and the readout at the frequency of the second harmonic. The dependences of the diffraction efficiency of the dynamic holograms formed at four-wave $(a)$ and sixwave $(b)$ interaction versus the intensity of the pumping waves are shown in Fig. 3 by solid lines. The calculations are conducted for the nonlinearity parameter $\alpha=$ $10^{-10} \mathrm{~W}^{-1} \mathrm{~cm}^{-2}$ and the ratio $a_{T} / \alpha=1000$, matching the experimental setup. The theoretical dependences are provided with the correction coefficients $1 / 4$ for the four-wave interaction and $2 / 3$ for the six-wave interaction. These coefficients account for the noninstantaneous and nonlocal character of the heat release and the dynamic gratings formation processes, as well as for the fact that the solution of system (2), (3) provides the value of the diffraction efficiency at the end of the pulse, while the experiment measured the diffraction efficiency averaged over the entire pulse.

\section{CONCLUSIONS}

The schemes of the frequency nondegenerated fourand six-wave interactions described above present an interest from the perspective of the frequency transformation of the coherent images, e.g., visualization of the IR images, as well as for the transformation of the phase of the diffracted wave. In the case of the six-wave interaction, a reversal of the wave front $\left(\varphi_{D}=-2 \varphi_{S}\right)$ is realized and the condition of the phase synchronization $\mathbf{k}_{D}=2 \mathbf{k}_{1}-2 \mathbf{k}_{S}+\mathbf{k}_{2}$ at the opposite directions of the reference and readout wave propagation $\mathbf{k}_{2}=-2 \mathbf{k}_{1}$ is automatically satisfied $\left(\mathbf{k}_{D}=-2 \mathbf{k}_{S}\right)$. In the case of the four-wave interaction, the preservation of the phase modulation $\left(\varphi_{D}=-\varphi_{S}\right)$ with the increase in the frequency $\left(\omega_{D}=2 \omega\right)$ means smoothing of the spatial structure of the wave front. The phase synchronization condition for the four-wave interaction $\mathbf{k}_{D}=\mathbf{k}_{1}-\mathbf{k}_{S}+\mathbf{k}_{2}$ in this case is satisfied for the predefined direction of the flat signal wave propagation, limiting the width of the angular spectrum of the restored image.

\section{REFERENCES}

1. Martin, G. and Hellwarth, R.W., Appl. Phys. Lett., 1979, vol. 34, p. 371.

2. Berezinskaya, A.M., Dukhovnyi, A.M., and Stasel'ko, D.I., Zh. Tekh. Fiz., 1983, vol. 53, no. 3, p. 499 [Tech. Phys. (Engl. Transl.), vol. 28, no. 3, p. 311].

3. Tolstik, A.L., Mnogovolnovye vzaimodeistviya $v$ rastvorakh slozhnykh organicheskikh soedinenii (Multiwave Interactions in Solutions of Complex Organic Compounds), Minsk: Izd-vo BGU, 2002.

4. Rubanov, A.S., Tolstik, A.L., Karpuk, S.M., and Ormachea, O., Opt. Commun., 2000, vol. 181, p. 183.

5. Ormachea, O. and Tolstik, A.L., Izv. Akad. Nauk, Ser. Fiz., 2005, vol. 69, no. 8, p. 1144.

6. Kabanov, V.V., Rubanov, A.S., and Tolstik, A.L., Kvantovaya Elektron. (Moscow), 1988, vol. 15, p. 1681.

7. Karpuk, S.M., Rubanov, A.S., and Tolstik, A.L., Kvantovaya Elektron. (Moscow), 1997, vol. 24, p. 52.

8. Charra, F. and Nunzi, J.-M., J. Opt. Soc. Am. B, 1991, vol. 8, p. 570. 